\title{
A novel method to identify differential pathways in uterine leiomyomata based on network strategy
}

\author{
HUI-LING WANG ${ }^{1}$, JING LIU ${ }^{2}$ and ZHAO-MIN QIN ${ }^{3}$ \\ ${ }^{1}$ First Gynecological Ward; ${ }^{2}$ Department of Health Checkup, Binzhou People's Hospital, Binzhou, \\ Shandong 256610; ${ }^{3}$ Department of Nursing, Shandong Medical College, Jinan, Shandong 250002, P.R. China
}

Received January 6, 2016; Accepted June 2, 2017

DOI: $10.3892 / \mathrm{ol} .2017 .6928$

\begin{abstract}
The aim of the present study was to identify differential pathways in uterine leiomyomata (UL) using a novel method based on protein-protein interaction networks and pathway analysis. The pathway networks were constructed by examining the intersections of the Reactome database and the Search Tool for the Retrieval of Interacting Genes/proteins (STRING) protein-protein interaction (PPI) networks. The Objective network was defined as the differential expressed genes (DEGs) associated with the interactions identified by STRING. Topological centrality (degree) analysis was performed for the Objective network to explore the hub genes and hub networks. Subsequent to isolating the intersections between the Pathway and Objective networks, randomization tests were conducted to identify the differential pathways. There were 559,598 interactions in the Pathway networks. A total of 657 genes with 3,835 interactions were mapped in the Objective network, which included 20 hub genes. It was identified that 358 pathways demonstrated interaction with the Objective network, such as Signal Transduction, Immune System and Signaling by G-protein-coupled receptor (GPCR). By accessing the randomization tests, P-values of these pathways were close to 0 , which indicated that they were significantly different. The present study successfully identified differential pathways (such as signal transduction, immune system and signaling by GPCR) in UL, which may be potential biomarkers in the detection and treatment of UL.
\end{abstract}

\section{Introduction}

Uterine leiomyomata (UL), a benign neoplasm deriving from the myometrial compartment of the uterus, is the most widespread gynecological problem in females (1). The common

Correspondence to: Dr Zhao-Min Qin, Department of Nursing, Shandong Medical College, 5460 South Second Ring Road, Jinan, Shandong 250002, P.R. China

E-mail: zhaominqin2015@yeah.net

Key words: uterine leiomyomata, pathway, network, interactions, count symptoms associated with UL are pelvic pain, discomfort, menstrual disorders and infertility (2). Surgery is the primary treatment modality, and tumors are often resistant to chemotherapy and radiation therapy (3). To date, adjuvant therapy has not demonstrated a significant survival advantage (3). Although surgical staging and nomograms may assist in predicting clinical outcome, the 5-year survival rate for uterus-confined disease remains $<50 \%$ (4). Understanding the molecular biology of UL may provide additional prognostic and therapeutic insights.

With the advances of high-throughput experimental technologies, these have been applied to explore the diagnostic gene signatures and biological processes of human diseases (5), which provide novel insights into the underlying biological mechanisms of UL. Microarray experiments have revealed that fibroid development may be due to abnormal tissue repair and an altered extracellular matrix (6). The levels of the inflammatory cytokine transforming growth factor- $\beta$ (TGF- $\beta$ ) were increased 3 -fold in fibroid tissue relative to myometrium $(7,8)$. However, a number of investigations on UL pathogenesis lack physiological relevance due to these studies being solely based on a number of individual genes and cell lines (9). Molecular pathways underlying UL development and growth acceleration are largely unknown, and the majority of previous results have stemmed from studying recurrent cytogenetic abnormalities identified among the $40 \%$ of abnormal UL (10), and gene expression profiles may be an additional good choice for research.

A variety of methods have been developed for the analysis of gene expression microarray data, but a small number of methods exist for using these data to quantify the interrelated behavior of genes within a gene interaction network (11). Even though the incidence of tumor is hypothesized to be closely associated with the abnormal expression of numerous genes, the studies on differential expressed genes (DEGs) is inadequate and there is a lot of work required to fully realize the potential of these DEGs. Therefore, studies investigating gene interactions are essential, as these interactions serve important roles in biological processes for cancer development (12). Previously, network-based approaches utilizing information concerning the interactions between gene pairs have emerged as powerful tools for the systematic understanding of the molecular mechanisms underlying biological processes important for cancer development, and several algorithms 
have been developed to study these biological networks. Barter et al (13) performed a comparative analysis and identified that the network-based method was more stable compared with single-gene and gene-set methods. However, there are a small number of studies identifying differential pathways dependent on network-based approaches $(11,14)$.

Therefore, in the present study, a novel method to identify differential pathways in UL based on gene interaction networks and pathway analysis was proposed. To achieve this, the primary step was to construct networks (Pathway, Objective and Hub networks), and analyzed their topological properties. Subsequently, the intersections between Pathway and Objective networks, and between Pathway and Hub networks, were isolated and randomization tests were performed to identify differential pathways in UL. This novel method may be an efficient supplement for identifying differential pathways.

\section{Materials and methods}

The primary component of this novel method consisted of Pathway network identification, Objective network construction, Hub network extraction and differential pathway evaluation. This method used to identify differential pathways is presented in Fig. 1.

Pathway network identification. Networks may provide new insights for mining unknown connections in incomplete networks. Although the data of large-scale protein interactions are accumulated with the development of high throughput testing technology, a certain number of significant interactions are not tested (14). This type of difficulty may be resolved to a certain extent by utilizing sub-networks of the complex network (15). Therefore, in the present study, pathway networks were identified by exploring the interactions of pathway-enriched genes with the global human protein-protein interaction (PPI) network from the Search Tool for the Retrieval of Interacting Genes/proteins (STRING) database (string-db. org; accessed August 24, 2015) (16). The pathway enriched genes originated from the Reactome pathway database (reactome.org; accessed July 13, 2015), which is a manually curated open-data resource of human pathways and reactions (17).

\section{Objective network construction}

Data collection and pretreatment. A total of two gene expression profiles [E-GEOD-18096 (18) and E-GEOD64763 (19)] for UL and normal controls were collected from ArrayExpress database (http://www.ebi.ac.uk/ arrayexpress/). E-GEOD-18096, which presented on the A-AFFY-44-Affymetrix GeneChip Human Genome U133 Plus 2.0 (HG-U133_Plus_2) platform, comprised $18 \mathrm{Ul}$ samples and 9 normal controls. E-GEOD-64763 existed on the A-AFFY-37-Affymetrix GeneChip Human Genome U133A 2.0 [HG-U133A_2] platform and consisted of $25 \mathrm{Ul}$ samples and 29 normal controls. In all, there were a total of 43 U1 samples and 38 normal controls in the two gene expression profiles.

Pretreatment for microarray expressions was performed to control the quality at probe level. The preprocess included four standard procedures: i) Background correction (20); ii) normalization (21); iii) probe correction (22); and

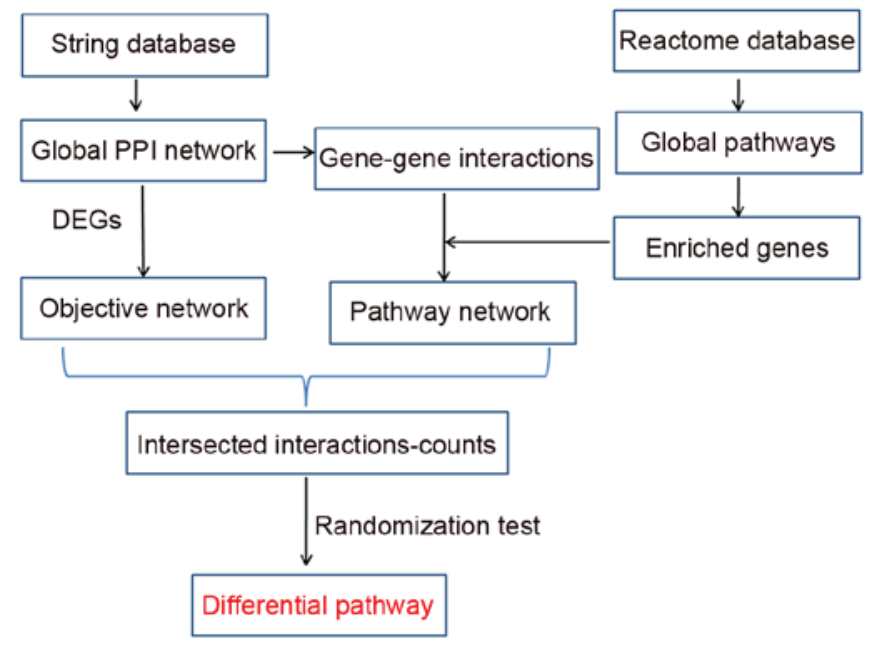

Figure 1. Method of identifying differential pathways between UL and normal controls. PPIs, protein-protein interactions; DEGs, differentially expressed genes; STRING, Search Tool for the Retrieval of Interacting Genes/proteins.

iv) summarization (20). The preprocessed probe-level dataset in CEL formats were converted into expression measures, and then screened by the feature filter method of gene filter package (23). Finally, a total of 20,102 and 12,493 genes were obtained from the E-GEOD-18096 and E-GEOD-64763 profiles, respectively. Subsequently, the empirical Bayes method in inSilicoMerging package version 1.15 .0 was utilized to merge the two preprocessed gene expression profiles into a single group (24) which included 12,493 genes for additional analysis.

DEGs detection. DEGs between UL and normal controls were identified using the linear models for microarray data (Limma) package version 3.30.0 (University of California, Berkeley, CA, USA) (25). All genes were manipulated with t test and F test, and then Linear fit, empirical Bayes statistics and false discovery rate correction were performed to the data by using lmFit function (26). DEGs were identified for additional study with the threshold of $\mathrm{P}<0.05$ and $\log$ FoldChangel $>2$.

Objective network construction. Certain significant genes may not be identifiable through their own behavior, but their changes are quantifiable when considered in conjunction with other genes, such as in a network (27). In the present study, a human PPI dataset from STRING as utilized to capture interactions among DEGs. The interactions were visualized by Cytoscape version 3.1.0 (Institute for Systems Biology, San Diego, CA, USA), and a PPI network was formed, which was defined as the Objective network. Cytoscape is a free software package for visualizing, modeling and analyzing the integration of bimolecular interaction networks with high-throughput expression data and other molecular states (28).

Hub network extraction. One of the fundamental problems in network analysis is to determine the importance of a particular node or an interaction between two nodes in a network, and quantifying centrality and connectivity assists in identifying portions of the network that may serve notable roles (29). In the present study, the biological importance of genes was characterized based on the Objective network using indices 
of topological centrality, degree centrality. The genes at the $\geq 97 \%$ quantile distribution in the significantly perturbed networks were defined as hub genes. In addition, the network, which was composed of hub genes and their interactions, was denoted as a hub network.

'Degree' quantifies the local topology of each gene by summing up the number of its adjacent genes $(j)$, and provides a simple count of the number of interactions of a given node (30). The degree $C_{\mathrm{D}}(v)$ of a node $v$ was calculated as following:

$$
C_{D}(v)=\sum_{j} a_{v j}
$$

In addition, the association between the number of genes and degree distribution was analyzed, and the fitting coefficient $\mathrm{R}^{2}$ of the power-law of the Objective network was detected, due to the fact that PPI networks in general are modular and scale-free, which meant that the network exhibited power-law (or scale-free) degree distributions $(31,32)$. The Network Analyzer 2.7 (Institute for Systems Biology) plugin in Cytoscape 3.1.0 was used for the evaluation of the topological parameters.

Differential pathways evaluation. The pathway, objective and hub networks were constructed, but selecting differential pathways based on the three kinds of networks was challenging. To overcome the problem, the intersections of the interactions between the Pathway and Objective networks, and between the Pathway and Hub networks were identified, and the quantity of intersected interactions was denoted as a 'count'. Subsequently, randomization tests were employed to determine the P-value of each pathway from the intersected interactions.

Randomization tests provide a general means of constructing tests that control size in finite samples whenever the distribution of the observed data exhibits symmetry under the null hypothesis (33). Let $T(X)$ be a real-valued test statistic such that large values provide evidence against the null hypothesis. Ordering $T^{(1)}(X) \leq T^{(2)}(X) \leq \cdots \leq T^{(M)}(X)$, denoting $k=M(1-\lambda)$ and define:

$$
\begin{aligned}
& M^{+}(X)=\left|\left\{1 \leq j \leq M: T^{(j)}(X)>T^{(k)}(X)\right\}\right| \\
& M^{0}(X)=\left|\left\{1 \leq j \leq M: T^{(j)}(X)=T^{(k)}(X)\right\}\right|
\end{aligned}
$$

Using this notation, the randomization tests were performed according to the following formulas (34):

$$
\begin{gathered}
\varphi(X)= \begin{cases}1 & \text { if } T(X)>T^{(k)}(X) \\
a(X) & \text { if } T(X)=T^{(x)}(X) \\
0 & \text { if } T(X)<T^{(k)}(X)\end{cases} \\
\text { Where } a(X)=\frac{M \lambda-M^{+}(X)}{M^{0}(X)}
\end{gathered}
$$

For any $\lambda \varepsilon(0,1), \phi(X)$ defined in the formula satisfied $\mathrm{P}[\phi(X)]=\lambda$. In the present study, $T(X)$ represented random networks that comprised intersected interactions, $\phi(X)$ stood for each pathway and $\mathrm{P}$ represented the significance of the pathway. If $\mathrm{P}<0.05$, this pathway was considered to be a differential pathway compared with normal controls.

\section{Results}

Pathway network. There were 787,896 interactions in the human STRING PPI network, while 1,675 human pathways were identified in the Reactome database. Interactions between pathway-enriched genes were extracted from the STRING database. A total of 559,598 gene-gene interactions were obtained, which formed a Pathway network. The 559,598 interactions may contain reduplicative interactions that were as a result of repeated enrichments of one interaction; one interaction was probably enriched in $\geq 2$ pathways.

Objective network construction and analysis. A total of 903 DEGs between patients with UL and normal controls were identified using the Limma package with thresholds of $\mathrm{P}<0.05$ and $\mid \log$ FoldChangel $>2$. When inputting these DEGs into the STRING database, 3,835 gene-gene interactions were obtained. Using Cytoscape, 657 genes with 3,835 interactions were mapped into the Objective network (Fig. 2). To additionally investigate the importance of individual genes in the bjective network, degree centrality analysis was conducted, and the degree distribution is presented in Fig. 3. The network analysis demonstrated that the Objective network presented a scale-free property whose degree distribution followed a power law $\left(y=a x^{b}\right.$, where $\left.a=117.0, b=-0.775\right)$ with the fitting coefficients $\mathrm{R}^{2}\left(\mathrm{R}^{2}=0.956\right)$.

Hub network extraction. In the present study, the genes in the $\geq 97 \%$ quantile distribution of 'degree' in the Objective network were defined as hub genes. In addition, the degree was calculated by summing up the number of adjacent genes. Consequently, a total of 20 hub genes were evaluated: Jun proto-oncogene, AP-1 transcription factor subunit (degree $=125$ ), epidermal growth factor receptor (degree=113), Fos proto-oncogene, AP-1 transcription factor subunit (degree $=108$ ), interleukin (IL)-6 (degree=85), cyclin D1 (degree=77), matrix metalloproteinase 9 (degree $=72$ ), intercellular adhesion molecule 1 (degree=68), early growth response 1 (degree=67), vascular endothelial growth factor A (degree=64), protein tyrosine phosphatase, receptor type C (degree $=60)$, KIT proto-oncogene receptor tyrosine kinase $($ degree $=59)$, peroxisome proliferator activated receptor gamma (degree $=55)$, toll like receptor 4 (degree $=51)$, topoisomerase $($ DNA) II $\alpha($ degree $=51)$, serpin family $\mathrm{E}$ member 1 (degree=50), cluster of differentiation $(C D) 44$ (degree=48), CD40 (degree=47), Acetyl-CoA carboxylase $\beta$ (degree=47), PH domain and leucine rich repeat protein phosphatase $1($ degree $=47)$ and Ras-related C3 botulinum toxin substrate 2 (Rho family, small GTP binding protein Rac2) (degree=47). The network, which was composed of hub genes and their interactions, was denoted as the Hub network, which was also the sub-network of the Objective network. Fig. 4 was the largest of the Hub networks, which included 10 hub genes.

Differential pathway identification. In the present study, randomization tests were implemented to identify differential pathways of UL based on the common interactions between the Pathway networks and the Objective network, and Pathway networks and Hub networks. During the examination of the intersections between the Pathway networks and Objective network, it was revealed that 358 pathways demonstrated interactions with the Objective network, but the numbers of interactions for the different pathways were markedly different, and listed counts $\geq 20$ in Table I. 'Count' signifies 


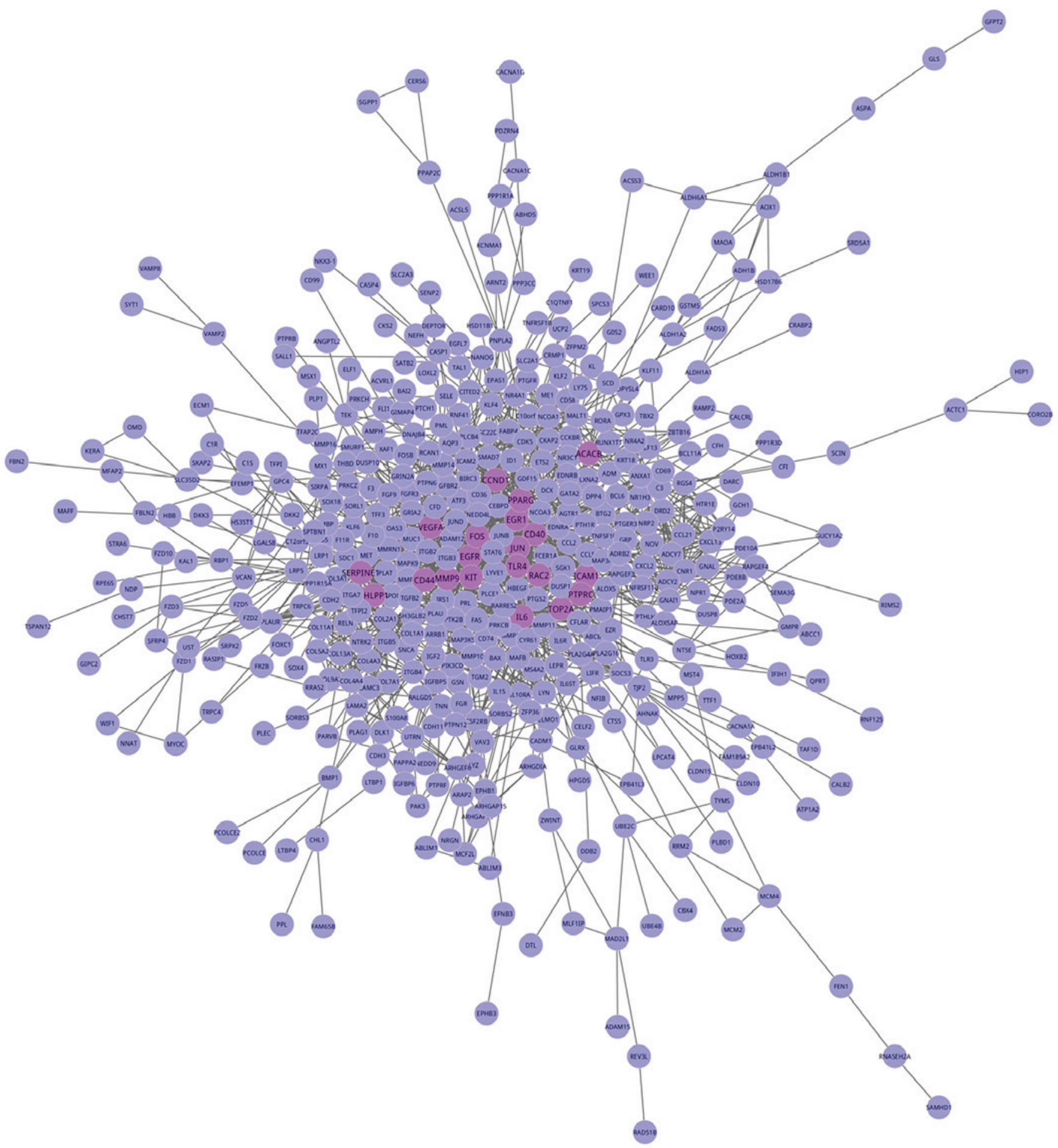

Figure 2. Objective network. Nodes represented genes, and lines between two nodes represent gene-gene interactions and the pink nodes are hub genes, which were defined as exhibiting $\geq 97 \%$ degree distribution. Purple nodes are genes.

the quantity of intersecting interactions. The top five pathways were signal transduction, with a count of 100, extracellular matrix organization, with a count of 87 , immune system, with a count of 81 , signaling by GPCR, with a count of 79 and GPCR downstream signaling, with a count of 60 . A total of $28.2 \%$ of the 358 pathways belonged to signal type pathways.

Concurrently, a total of 162 pathways interacted with the Hub networks, and Table II summarizes the pathways with counts $\geq 10$. Immune system, signal transduction, innate immune system, hemostasis and signaling by GPCR were the top five in descending order, with counts of 49, 42, 29, 19 and 19, respectively. The 162 pathways were all involved in the intersections between the Pathway networks and the Objective network. It also validated the feasibility and accuracy of this method in identifying the differential pathways in UL.

If $\mathrm{P}<0.05$, the pathway was considered to be a differential pathway. Notably, the majority of the P-values were close to or equal to 0 , which suggested that these pathways were 
Table I. Intersections ( $\geq 20$ ) between the pathway and objective networks.

\begin{tabular}{lr}
\hline Pathway & Count \\
\hline Signal transduction & 100 \\
Extracellular matrix organization & 87 \\
Immune system & 81 \\
Signaling by GPCR & 79 \\
GPCR downstream signaling & 60 \\
Innate immune system & 49 \\
G $\alpha$ (i) signaling events & 34 \\
GPCR ligand binding & 34 \\
Class A/1 (rhodopsin-like receptors) & 31 \\
Collagen formation & 31 \\
Assembly of collagen fibrils and other & 30 \\
multimeric structures & \\
Cytokine signaling in immune system & 25 \\
Developmental biology & 25 \\
Hemostasis & 24 \\
Metabolism of lipids and lipoproteins & 22 \\
Gastrin-cyclic adenosine 5'-monophosphate & 21 \\
response element binding protein signaling & \\
pathway via protein kinase C and & 21 \\
mitogen-activated protein kinase & \\
Metabolism & \\
Gene expression & \\
\hline
\end{tabular}

GPCR, G-protein-coupled receptor.

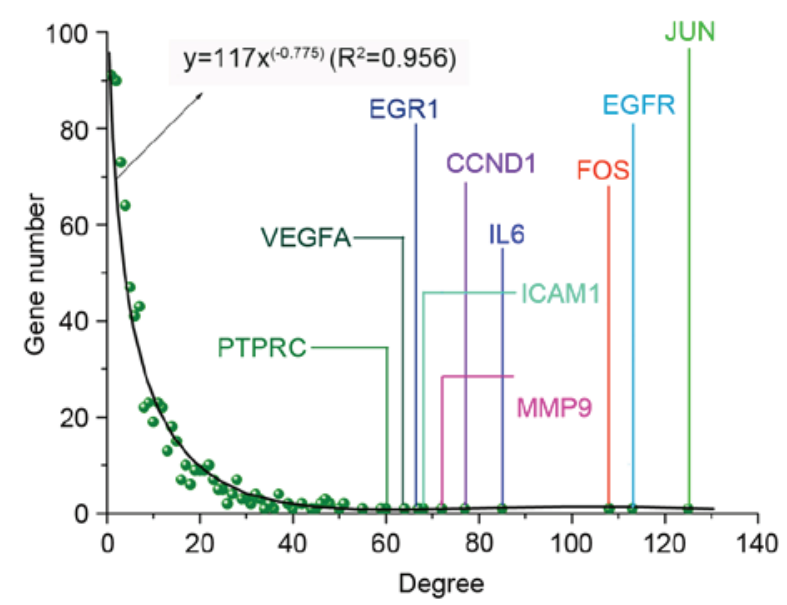

Figure 3. Gene degree distribution in the objective network. The objective network was a scale-free network whose degree distribution followed a power law $\left(\mathrm{y}=\mathrm{ax}{ }^{\mathrm{b}}\right.$, where $\left.\mathrm{a}=117.0, \mathrm{~b}=-0.775\right)$ with the fitting coefficient $\left(\mathrm{R}^{2}=0.956\right)$. PHLPP1, PH domain and leucine rich repeat protein phosphatase 1; VEGFA, vascular endothelial growth factor A; EGR1, early growth response 1; $C C N D 1$, cyclin D1; IL6, interleukin 6; $M M P 9$, matrix metalloproteinase 9; ICAM1, intercellular adhesion molecule 1; FOS, Fos proto-oncogene, AP-1 transcription factor subunit; $E G F R$, epidermal growth factor receptor; JUN, Jun proto-oncogene, AP-1 transcription factor subunit.

significantly differential. Due to the similar P-values of the differential pathways, the count may be an additional measure to evaluate the significance of pathways. The higher of count,
Table II. Intersections $(\geq 10)$ between the pathway and hub networks.

\begin{tabular}{lc} 
Pathway & Count \\
\hline Immune system & 49 \\
Signal transduction & 42 \\
Innate immune system & 29 \\
Hemostasis & 19 \\
Signaling by GPCR & 19 \\
Cytokine signaling in immune system & 17 \\
Developmental biology & 17 \\
Extracellular matrix organization & 17 \\
Fc $\varepsilon$ receptor signaling & 13 \\
TLR cascades & 13 \\
Activated TLR4 signaling & 12 \\
TLR4 cascade & 12 \\
Axon guidance & 11 \\
MyD88-independent TLR3/TLR4 cascade & 10 \\
TLR3 cascade & 10 \\
TIR-domain-containing adapter-inducing & 10 \\
interferon- $\beta$-mediated TLR3/TLR4 signaling &
\end{tabular}

TLR, Toll-like receptors.

the closer the association between the pathway and UL, such as signal transduction, with a count of 100 .

\section{Discussion}

To identify the differential pathways of UL, a novel method including Pathway, Objective and Hub networks, was proposed. The topological properties of gene interaction networks have been studied widely (30). It has been indicated that gene interaction networks also have scale-free properties $(35,36)$, which are typical of biological networks. Featherstone and Broadie (37) demonstrated that the scale-free distribution of gene degrees in network assisted organisms in developing resistance to the deleterious effects of mutation. Similar architecture was also identified in the gene co-expression networks of gastric cancer (38). In the present study, a novel network-based method was produced, in which the objective network was revealed to be an evidently scale-free network, whose node degree distribution followed a power law with the fitting coefficient, which validated the reliability and feasibility of the network-based method.

A total of 358 differential pathways were identified, based on networks and randomization tests with $\mathrm{P}<0.05$, for example, signal transduction, immune system and signaling by GPCR. In addition, the differential pathways obtained from Hub networks were all involved in these 358 pathways, attributing to the Hub network presented as a sub-network of objective network, and confirmed the repeatability of the present study.

In detail, $28.2 \%$ of the 358 differential pathways were associated with signaling, for example: Signal transduction and signaling by GPCR. Signal transduction occurs when an extracellular signaling molecule activates a specific receptor 


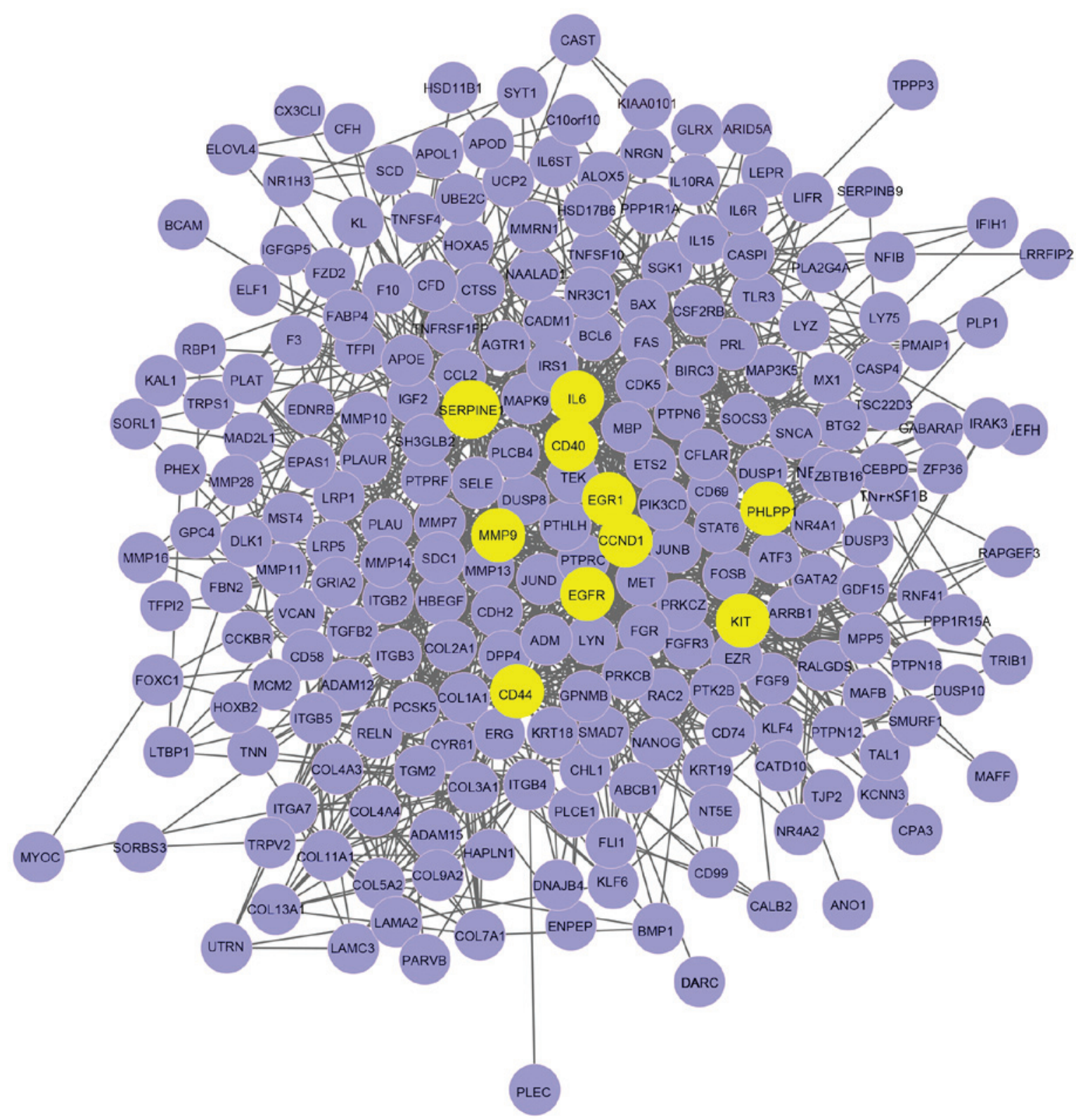

Figure 4. Hub network. There were 10 hub genes in the network; EGFR, IL6, CCND1, MMP9, EGR1, KIT, SERPINE1, CD44, CD40 and PHLPP1. Nodes represented genes, and lines between two nodes represent for gene-gene interactions. Yellow nodes are hub genes, purple nodes are genes. EGFR, epidermal growth factor receptor; IL6, interleukin 6; CCND1, cyclin D1; $M M P$ 9, matrix metalloproteinase 9; EGR1, early growth response 1; KIT, KIT proto-oncogene receptor tyrosine kinase; SERPINE1, serpin family E member 1; CD, cluster of differentiation; PHLPP1, PH domain and leucine rich repeat protein phosphatase 1.

located on the cell surface or inside the cell, which in turn triggers a biochemical chain of events inside the cell, creating a response (39). Depending on the cell, the response alters the metabolism, shape, gene expression of the cell, or ability of the cell to divide: Dysregulation of these processes may lead to cancer (40). It had been suggested that certain microbial molecules, such as viral nucleotides and protein antigens, may elicit an immune system response against invading pathogens, mediated by signal transduction processes (41). Gene activations and alterations in metabolism were examples of cellular responses to extracellular stimulation that required signal transduction (42). The mitogen-activated protein kinase/extracellular-signal related protein kinase pathway couples intracellular responses to the binding of growth factors to cell surface receptors, and its activation promoted cell division and numerous forms of cancer are associated with aberrations in it, such as UL (43). Therefore, signal transduction serves a significant role in UL development.

The immune system is a system involving a number of biological structures and processes within an organism that protects against disease. To function properly, it detects a wide variety of pathogens (from viruses to parasitic worms) and distinguishes them from the organism's own healthy tissue (44). In a number of species, the immune system may be classified into subsystems, such as the innate immune system versus the adaptive immune system, or humoral immunity versus cell-mediated immunity. 'Innate immune system' was an additional important differential pathway in the present study. The present study indicated that UL development may be triggered, at least in part, by a chronically-active inflammatory immune system. The concept of inflammation actually serves a theory of fibroid development based on an altered response to noxious stimuli; possibly tissue 
injury from extravasated menstrual blood into the myometrium, or hypoxia leading to altered tissue repair and fibroids (45). It had been demonstrated that leiomyoma formation may be acquired through investigation of immune system (46). Complex interactions between the endocrine and immune systems govern the key endometrial events, and inflammatory pathway dysfunction was present in the endometria of women with endometriosis and uterine fibroids (47). Santulli et al (48) revealed that IL-33 was released as a danger signal, alerting the immune system following endogenous stimulation, and elevated serum IL-33 levels were associated with the existence of UL (48). The present study identified that IL6 was a hub gene in UL, and perhaps also took part in the signal activity and served a critical role in UL.

In conclusion, the present study successfully identified differential pathways (such as signal transduction, immune system and signaling by GPCR) in UL, which may provide potential insights into the detection and treatment of UL.

\section{References}

1. Islam MS, Protic O, Giannubilo SR, Toti P, Tranquilli AL, Petraglia F, Castellucci M and Ciarmela P: Uterine leiomyoma: Available medical treatments and new possible therapeutic options. J Clin Endocrinol Metab 98: 921-934, 2013.

2. Buttram VC Jr and Reiter RC: Uterine leiomyomata: Etiology, symptomatology, and management. Fertil Steril 36: 433-445, 1981.

3. Gallup DG, Blessing JA, Andersen W and Morgan MA; Gynecologic Oncology Group Study: Evaluation of paclitaxel in previously treated leiomyosarcoma of the uterus: A gynecologic oncology group study. Gynecol Oncol 89: 48-51, 2003.

4. Zivanovic O, Jacks LM, Iasonos A, Leitao MM Jr, Soslow RA, Veras E, Chi DS, Abu-Rustum NR, Barakat RR, Brennan MF, et al: A nomogram to predict postresection 5-year overall survival for patients with uterine leiomyosarcoma. Cancer 118: 660-669, 2012

5. Jordán F, Nguyen TP and Liu WC: Studying protein-protein interaction networks: A systems view on diseases. Brief Funct Genomics 11: 497-504, 2012.

6. Koohestani F, Braundmeier AG, Mahdian A, Seo J, Bi J and Nowak RA: Extracellular matrix collagen alters cell proliferation and cell cycle progression of human uterine leiomyoma smooth muscle cells. PLoS One 8: e75844, 2013.

7. Halder SK, Goodwin JS and Al-Hendy A: 1,25-Dihydroxyvitamin D3 reduces TGF-beta3-induced fibrosis-related gene expression in human uterine leiomyoma cells. J Clin Endocrinol Metab 96: E754-E762, 2011.

8. Moore AB, Yu L, Swartz CD, Zheng X, Wang L, Castro L, Kissling GE, Walmer DK, Robboy SJ and Dixon D: Human uterine leiomyoma-derived fibroblasts stimulate uterine leiomyoma cell proliferation and collagen type I production, and activate RTKs and TGF beta receptor signaling in coculture. Cell Commun Signal 8: 10, 2010.

9. Lin CP, Chen YW, Liu WH, Chou HC, Chang YP, Lin ST, Li JM, Jian SF, Lee YR and Chan HL: Proteomic identification of plasma biomarkers in uterine leiomyoma. Mol Biosyst 8: 1136-1145, 2012.

10. Hodge JC, Kim TM, Dreyfuss JM, Somasundaram P, Christacos NC, Rousselle M, Quade BJ, Park PJ, Stewart EA and Morton CC: Expression profiling of uterine leiomyomata cytogenetic subgroups reveals distinct signatures in matched myometrium: Transcriptional profiling of the $t(12 ; 14)$ and evidence in support of predisposing genetic heterogeneity. Hum Mol Genet 21: 2312-2329, 2012.

11. Toyoshiba H, Yamanaka T, Sone H, Parham FM, Walker NJ, Martinez J and Portier CJ: Gene interaction network suggests dioxin induces a significant linkage between aryl hydrocarbon receptor and retinoic acid receptor beta. Environ Health Perspect 112: 1217-1224, 2004.

12. Toyoshiba H, Sone H, Yamanaka T, Parham FM, Irwin RD, Boorman GA and Portier CJ: Gene interaction network analysis suggests differences between high and low doses of acetaminophen. Toxicol Appl Pharmacol 215: 306-316, 2006.
13. Barter RL, Schramm SJ, Mann GJ and Yang YH: Network-based biomarkers enhance classical approaches to prognostic gene expression signatures. BMC Syst Biol 8 (Suppl 4): S5, 2014.

14. Nibbe RK, Chowdhury SA, Koyutürk M, Ewing R and Chance MR: Protein-protein interaction networks and subnetworks in the biology of disease. Wiley Interdiscip Rev Syst Biol Med 3: 357-367, 2011.

15. Wu Y, Jing R, Jiang L, Jiang Y, Kuang Q, Ye L, Yang L, Li Y and $\mathrm{Li} \mathrm{M}$ : Combination use of protein-protein interaction network topological features improves the predictive scores of deleterious non-synonymous single-nucleotide polymorphisms. Amino Acids 46: 2025-2035, 2014.

16. Szklarczyk D, Franceschini A, Wyder S, Forslund K, Heller D, Huerta-Cepas J, Simonovic M, Roth A, Santos A, Tsafou KP, et al: STRING v10: Protein-protein interaction networks, integrated over the tree of life. Nucleic Acids Res 43 (Database issue): D447-D452, 2015.

17. Croft D, Mundo AF, Haw R, Milacic M, Weiser J, Wu G, Caudy M, Garapati P, Gillespie M, Kamdar MR, et al: The Reactome pathway knowledgebase. Nucleic Acids Res 42 (Database issue): D472-D477, 2014.

18. Hodge JC, Kim TM, Dreyfuss JM, Somasundaram P, Christacos NC, Rousselle M, Quade BJ, Park PJ, Stewart EA and Morton CC: Expression profiling of uterine leiomyomata cytogenetic subgroups reveals distinct signatures in matched myometrium: Transcriptional profilingof the $\mathrm{t}(12 ; 14)$ and evidence in support of predisposing genetic heterogeneity. Hum Mol Genet 21: 2312-2329, 2012.

19. Barlin JN, Zhou QC, Leitao MM, Bisogna M, Olvera N, Shih KK, Jacobsen A, Schultz N, Tap WD, Hensley ML, et al: Molecular subtypes of uterine leiomyosarcoma and correlation with clinical outcome. Neoplasia 17: 183-189, 2015.

20. Irizarry RA, Bolstad BM, Collin F, Cope LM, Hobbs B and Speed TP: Summaries of Affymetrix Genechip probe level data. Nucleic Acids Res 31: e15, 2003.

21. Bolstad BM, Irizarry RA, Astrand M and Speed TP: A comparison of normalization methods for high density oligonucleotide array data based on variance and bias. Bioinformatics 19: 185-193, 2003.

22. Bolstad B: affy: Built-in processing methods, 2013.

23. Lee $\mathbf{J}$ and Kim DW: Efficient multivariate feature filter using conditional mutual information. Electron Lett 48: 161-162, 2012.

24. Taminau J, Meganck S and Lazar C: Using the inSilicoMerging package. http://www.bioconductor.org/packages//2.10/ bioc/vignettes/inSilicoMerging/inst/doc/inSilicoMerging.pdf. Accessed June 22, 2012.

25. Smyth GK: Linear models and empirical bayes methods for assessing differential expression in microarray experiments. Stat Appl Genet Mol Biol 3: Article3, 2004.

26. Diboun I, Wernisch L, Orengo CA and Koltzenburg M: Microarray analysis after RNA amplification can detect pronounced differences in gene expression using limma. BMC Genomics 7: 252, 2006.

27. Zhuang DY, Jiang L, He QQ, Zhou P and Yue T: Identification of hub subnetwork based on topological features of genes in breast cancer. Int J Mol Med 35: 664-674, 2015.

28. Smoot ME, Ono K, Ruscheinski J, Wang PL and Ideker T: Cytoscape 2.8: New features for data integration and network visualization. Bioinformatics 27: 431-432, 2011.

29. Bader DA and Madduri K: Parallel algorithms for evaluating centrality indices in real-world networks. In: Parallel Processing, 2006. ICPP 2006, Int Confer IEEE, Columbus, OH, pp539-550, 2006.

30. Haythornthwaite C: Social network analysis: An approach and technique for the study of information exchange. Lib Inf Sci Res 18: 323-342, 1996.

31. Ravasz E, Somera AL, Mongru DA, Oltvai ZN and Barabási AL: Hierarchical organization of modularity in metabolic networks. Science 297: 1551-1555, 2002.

32. Rifai $\mathrm{N}$ and Ridker PM: Proposed cardiovascular risk assessment algorithm using high-sensitivity C-reactive protein and lipid screening. Clin Chem 47: 28-30, 2001.

33. Canay IA, Romano JP and Shaikh AM: Randomization tests under an approximate symmetry assumption. Econometrica 85: 1013-1030, 2017.

34. Ibragimov R and Müller UK: t-Statistic based correlation and heterogeneity robust inference. J Business Eco Stat 28: 453-468, 2010.

35. Jordan IK, Mariño-Ramírez L, Wolf YI and Koonin EV: Conservation and coevolution in the scale-free human gene coexpression network. Mol Biol Evol 21: 2058-2070, 2004. 
36. van Noort V, Snel B and Huynen MA: The yeast coexpression network has a small-world, scale-free architecture and can be explained by a simple model. EMBO Rep 5: 280-284, 2004.

37. Featherstone DE and Broadie K: Wrestling with pleiotropy: Genomic and topological analysis of the yeast gene expression network. Bioessays 24: 267-274, 2002.

38. Aggarwal A, Guo DL, Hoshida Y, Yuen ST, Chu KM, So S, Boussioutas A, Chen X, Bowtell D, Aburatani $\mathrm{H}$, et al: Topological and functional discovery in a gene coexpression meta-network of gastric cancer. Cancer Res 66: 232-241, 2006.

39. Hynes NE, Ingham PW, Lim WA, Marshall CJ, Massagué J and Pawson T: Signalling change: Signal transduction through the decades. Nat Rev Mol Cell Biol 14: 393-398, 2013.

40. Cabal-Hierro L and Lazo PS: Signal transduction by tumor necrosis factor receptors. Cell Signal 24: 1297-1305, 2012.

41. Würstle ML, Laussmann MA and Rehm M: The central role of initiator caspase-9 in apoptosis signal transduction and the regulation of its activation and activity on the apoptosome. Exp Cell Res 318: 1213-1220, 2012.

42. Wynn ML, Merajver SD and Schnell S: Unraveling the complex regulatory relationships between metabolism and signal transduction in cancer. Adv Exp Med Biol 736: 179-189, 2012.

43. Makker A, Goel MM, Das V and Agarwal A: PI3K-Akt-mTOR and MAPK signaling pathways in polycystic ovarian syndrome, uterine leiomyomas and endometriosis: An update. Gynecol Endocrinol 28: 175-181, 2012.

44. Parham P: The Immune System. 4th Edition. Garland Science, New York, NY, 2014
45. Leppert P, Fouany $\mathrm{M}$ and Segars JH: Understanding uterine fibroids. In: Fibroids. Segars JH (ed). John Wiley \& Sons, Ltd., Oxford, 2013

46. Wegienka G, Baird DD, Cooper T, Woodcroft KJ and Havstad S Cytokine patterns differ seasonally between women with and without uterine leiomyomata. Am J Reprod Immunol 70: 327-335, 2013.

47. Maybin JA, Critchley HO and Jabbour HN: Inflammatory pathways in endometrial disorders. Mol Cell Endocrinol 335: 42-51, 2011.

48. Santulli P, Even M, Chouzenoux S, Millischer AE, Borghese B, de Ziegler D, Batteux F and Chapron C: Profibrotic interleukin-33 is correlated with uterine leiomyoma tumour burden. Hum Reprod 28: 2126-2133, 2013. 